\title{
Traumatismo cranioencefálico leve: uma breve revisão
}

\section{Mild Traumatic Brain Injury: A Brief Review}

\author{
Renan Ribeiro Teixeira ${ }^{1}$ Almir Ferreira de Andrade ${ }^{2}$ Manoel Jacobsen Teixeira ${ }^{1}$ \\ Eberval Gadelha Figueiredo ${ }^{1}$
}

${ }^{1}$ Faculdade de Medicina, Universidade de São Paulo, São Paulo, SP, Brazil

2 Emergency Neurosurgery Service, Hospital das Clínicas, Faculdade de Medicina, Universidade de São Paulo, São Paulo, SP, Brazil

Address for correspondence Renan Ribeiro Teixeira, Faculdade de Medicina, Universidade de São Paulo, São Paulo, Brazil (e-mail: renan.ribeiro.teixeira@usp.br).

Arq Bras Neurocir

\section{Resumo \\ Palavras-Chave \\ - traumatismo encefálico \\ - epidemiologia \\ - diagnóstico \\ - prognóstico}

Considerado a principal causa de invalidez, morte e consumo de recursos, o traumatismo cranioencefálico (TCE) pode ser definido como uma alteração da função normal do cérebro causada por colisão ou movimentos bruscos na cabeça, ou ainda por um ferimento penetrante. Os TCEs são classificados como leves, moderados ou graves. No caso dos traumas leves, há uma grande variedade em sua classificação, que pode ser feita com base no uso da escala de Glasgow entre 13 e 15, em alterações da consciência, em amnésia, na nona revisão da Classificação Estatística Internacional de Doenças e Problemas Relacionados com a Saúde (CID-9), e até na escala abreviada de lesões (EAL). Devido a sua importância (corresponde a 75\% de todos os TCEs e, segundo o Centers for Disease Control and Prevention [CDC], há aproximadamente 500 novos casos por 100 mil pessoas por ano), este trabalho faz uma revisão breve apontando a epidemiologia, as formas de diagnóstico e os possíveis prognósticos. Assim, podemos entender melhor os traumas leves, pois este é um dos poucos distúrbios da medicina em que a classificação benigna é enganosa, e pode estar associada a complicações ao longo da vida.

Considered to be the main cause of invalidity and death, besides being extremely costly to the health care system, cranioencephalic trauma can be defined as an alteration of the normal brain function that can be caused by collisions, abrupt head movements or even penetrating injuries. Traumatic brain injuries (TBI) can be classified as mild, moderate or severe. In the case of mild TBI, there is a great variability in its classification, which can be based on the Glasgow coma scale (between 13 and 15), alterations in consciousness, amnesia, the International Classification of Diseases, Ninth Revision (ICD-9), and even on the abbreviated injury scale (AIS). Due to the importance of mild TBI (it corresponds to $75 \%$ of all TBIs and, according to the Centers for Disease Control and Prevention [CDC], there are $\sim 500$ new cases per every 100,000 persons per year), the present report consists of a brief review of the epidemiology, diagnosis and possible prognosis of this type of injury. Further understanding is paramount, since mild TBI is one of the few disorders in medicine in which the benign classification can be misleading and still be associated to further complications. received

December 2, 2016

accepted

December 13, 2016
DOI http://dx.doi.org/

10.1055/s-0037-1598610. ISSN 0103-5355.
Copyright $\odot$ by Thieme-Revinter

Publicações Ltda, Rio de Janeiro, Brazil
License terms

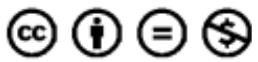




\section{Introdução}

O Center for Disease Control and Prevention (CDC) define traumatismo cranioencefálico (TCE) como uma alteração da função normal do cérebro causada por colisão ou movimentos bruscos na cabeça, ou ainda por um ferimento penetrante. $^{1} \mathrm{O}$ trauma cranioencefálico é a principal causa de invalidez, morte e consumo de recursos. Há dois tipos de lesões no TCE, denominadas primária e secundária (a lesão primária se dá por forças físicas inicialmente aplicadas no cérebro pelo impacto, e a lesão secundária ocorre ao longo de um período de horas ou dias após o trauma, resultando na ativação de vias diferentes, tais como a coagulação, a inflamação, a oxidação e a apoptose).

Após o TCE, devemos estar atentos aos seguintes sinais: período de perda ou diminuição da consciência; alguma perda de memória do evento imediatamente anterior (amnésia retrógada) ou após a lesão (amnésia pós-traumática); déficits neurológicos, como perda de força muscular, alteração da visão, alteração da linguagem e fala, diminuição da coordenação, diminuição da sensibilidade; e alguma alteração no estado mental no momento da lesão, como desorientação, dificuldade de concentração, confusão e pensamento retardado. ${ }^{2}$ Estudos mostram alguns sinais que indicam a necessidade de encaminhamento do paciente a um centro de referência: queda progressiva do nível de consciência e das funções neurocognitivas; convulsões; perda de consciência num período superior a 5 minutos; e aumento das dores de cabeça e afasias, o que indica uma condição de maior gravidade. ${ }^{3}$

Segundo a idade, os grupos de grande risco para TCE são as crianças de até 4 anos, adolescentes entre 15 e 19 anos, e os idosos acima de 65 anos. De acordo com o CDC, em pesquisa feita em salas de emergência, quedas compreendem $38 \%$ dos casos de TCE. Os acidentes de trânsito (acidentes com veículos, assim como acidentes envolvendo pedestres e ciclistas) contribuem para $16 \%$ dos casos; as contusões representam $20 \%$; já os assaltos, $11 \%$; e outras causas correspondem a $15 \%$ dos casos. ${ }^{4}$ Em 2015, uma revisão da literatura sobre a epidemiologia do TCE no Brasil trouxe algumas conclusões sobre a matéria, como o fato de os TCEs serem predominantes no sexo masculino, variando entre taxas de 2,2:1 a 7,6:1. ${ }^{5}$

O TCE pode se apresentar como uma lesão focal ou difusa, sendo que algumas pessoas podem apresentar ambas. A lesão focal é mais frequentemente decorrente de uma hemorragia. A lesão difusa do cérebro ocorre quando o mesmo sofre ações de forças de aceleração e desaceleração. 0 impacto na cabeça causado por um objeto poderá provocar uma lesão focal sobre o local do impacto no crânio ou no lado oposto. ${ }^{6}$ O TCE tem como forma mais comum a combinação de impactos e forças de aceleração/desaceleração, como as ocorridas em acidentes com veículos em alta velocidade. ${ }^{7}$ Além disso, forças externas podem causar movimentos cerebrais que levam à perda da integridade dos tecidos nervosos (ruptura dos axônios), ou que podem até mesmo causar fraturas dos ossos do crânio. Os lobos frontal e temporal são particularmente mais susceptíveis a este fenômeno por serem superfícies em que os tecidos podem ser facilmente lesados. Por esta razão, levantou-se a hipótese de que a suscetibilidade dos lobos frontotemporais são a base para os sintomas cognitivos e comportamentais comumente experimentados após um TCE. ${ }^{8}$

Devido a esta gama de lesões e apresentações clínicas das mais diversas, a classificação do TCE baseada em padrões ou tipos de lesões é importante para propormos tratamento e terapia em longo prazo. Entretanto, a complexidade do TCE e as limitações de ferramentas para avaliação tornam-se um desafio. ${ }^{1}$ Costumamos classificar o TCE em leve, moderado ou grave segundo a escala de Glasgow ( - Tabela 1$)^{9}$ registrada na sala de emergência, a duração da amnesia pós-traumática (perda de memória após o evento), e a duração da perda de consciência. ${ }^{4}$ Como apresentado também na - Tabela $\mathbf{2}$, segundo as diretrizes do Department of Veterans Affairs e do Department of Defense (VA/DoD) ${ }^{10}$ dos Estados Unidos, há classificações segundo a escala e outros fatores (duração da alteração do estado mental ou perda da consciência e duração da amnésia pós-traumática), sendo que estes não podem estar relacionados ao uso de medicamentos, álcool, intoxicação por drogas e falência dos órgãos. Exames de imagem também podem ser usados para identificar possíveis alterações, podendo ajudar na avaliação quanto à gravidade da lesão. ${ }^{4}$ Alguns estudos utilizam, além dessa ferramenta de classificação, a escala abreviada de lesões (EAL), baseada em seis pontos que demonstram o risco de mortalidade, variando de " 1 " para lesão mínima, enquanto " 6 " demonstra que o paciente não sobreviveu. Existe uma relação entre a classificação e a EAL, como mostrado na - Tabela $3 .^{11}$ Sendo

Tabela 1 Escala de coma de Glasgow

\begin{tabular}{|l|l|}
\hline & Score \\
\hline Abertura dos olhos & \\
\hline Espontânea & 4 \\
\hline Ao chamado & 3 \\
\hline Há dor & 2 \\
\hline Não abre & 1 \\
\hline Resposta motora & \\
\hline Normal & 6 \\
\hline Localiza estímulo doloroso & 5 \\
\hline Remove a dor & 4 \\
\hline Flexão anormal à dor & 3 \\
\hline Extensão anormal da dor & 2 \\
\hline Não responde & 1 \\
\hline Resposta Verbal & \\
\hline Conversa normal & 5 \\
\hline Fala desorientada & 4 \\
\hline Palavras sem coerência & 3 \\
\hline Sem palavras, apenas sons & 2 \\
\hline Não responde & 1 \\
\hline
\end{tabular}

Nota: Score: abertura dos olhos + resposta motora + resposta verbal $=$ de 3 a 15

Tabela copiada de Teasdale and jennett (1974). ${ }^{9}$ 
Tabela 2 Diretrizes para a prática clínica na classificação da gravidade de TCEs do VA/DoD ${ }^{10}$

\begin{tabular}{|l|l|l|l|}
\hline Critério & Leve & Moderado & Grave \\
\hline Exame de imagem & Normal & Normal ou anormal & Normal ou anormal \\
\hline Perda de consciência & $\leq 30 \mathrm{~min}$ & de 30 min a $24 \mathrm{~h}$ & $\geq 24 \mathrm{~h}$ \\
\hline Alteração da consciência/estado mental & $\leq 24 \mathrm{~h}$ & $\begin{array}{l}>24 \mathrm{~h} \\
\text { (gravidade baseada em outros critérios) }\end{array}$ \\
\hline Amnésia pós-traumática & $\geq 1 \mathrm{dia}$ & de $1 \mathrm{a} 7$ dias & $\geq 7$ dias \\
\hline $\begin{array}{l}\text { Escala de coma de Glasgow (melhor avaliação } \\
\text { feita nas primeiras 24 horas) }\end{array}$ & $13-15$ & $9-12$ & $<9$ \\
\hline
\end{tabular}

Abreviaturas: Dod, Department of Defense; TCE, traumatismo cranioencefálico; VA, Department of Veteran Affairs. Nota: Tabela copiada de Krainin et al (2001). ${ }^{3}$

Tabela 3 Critérios usados para classificar a gravidade do TCE

\begin{tabular}{|c|c|c|c|}
\hline & \multicolumn{3}{|c|}{ Gravidade do TCE } \\
\hline & Leve & Moderado & Grave \\
\hline Exame de imagem & Normal & Normal ou anormal & Normal ou anormal \\
\hline Perda de consciência & $\leq 30 \min$ & de $30 \min$ a $24 \mathrm{~h}$ & $\geq$ de $24 \mathrm{~h}$ \\
\hline Amnésia pós-traumática & $0-1$ dia & de 1 a 7 dias & $>7$ dias \\
\hline Escala de coma de Glasgow & $13-15$ & $9-12$ & $3-8$ \\
\hline Score da escala abreviada de lesões: cabeça & $1-2$ & 3 & $4-6$ \\
\hline
\end{tabular}

Abreviatura: TCE, traumatismo cranioencefálico.

Nota: Tabela copiada de Brasure et al (2012). ${ }^{11}$

assim, cada critério usado de forma isolada não pode permitir indicar a gravidade da lesão.

Krainin BM et $\mathrm{al}^{3}$ propuseram uma nova classificação do TCE. Primeiramente, as diferentes apresentações que se seguem ao trauma (seja em curto ou longo prazo) permitem a sua classificação em agudo ou crônico, sendo que os traumas agudos poderiam ser classificados por um sistema de graus que pode se modificar de acordo com a alteração dos sintomas (- Tabela 4). ${ }^{10}$ Essa nova classificação indica melhor a gravidade dos traumatismos, e permite eliminar a nomenclatura "leve", "moderado" e "grave," pois estes nomes às vezes não mostram com precisão a gravidade da lesão (traumas leves podem ter apresentação clínica grave, não compatível com o nome). Por fim, pela apresentação clínica inicial do TCE, não se podem prever os resultados em longo prazo. Para amenizar este paradoxo, na - Tabela 5 há a proposição de uma categorização que reflete o Diagnostic and Statistical Manual of Mental Disorders, quinta edição (DSM-5), da American Psychiatric Association (APA) ${ }^{12}$, e que descreve uma variedade de classificações subagudas e crônicas. Sendo assim, um paciente que apresente grau 3 no TCE agudo pode, em longo prazo, apresentar sintomas mínimos, e seu diagnóstico pode ser alterado para grau 1 no TCE crônico; o inverso também pode ser verdade. ${ }^{3}$

\section{Dificuldades de definição e patologia do TCE leve}

O TCE é reconhecido como entidade médica desde a época de Hipócrates. O termo concussão tem sido usado das mais

Tabela 4 Proposição de critério diagnóstico do TCE agudo

\begin{tabular}{|l|l|l|l|}
\hline Critério & Grau 1 & Grau 2 & Grau 3 \\
\hline Exame de imagem & Normal & Normal ou anormal & Normal ou anormal \\
\hline Perda da consciência & $\leq 30 \mathrm{~min}$ & de 30 min a 24 h & $>24 \mathrm{~h}$ \\
\hline Alteração da consciência/estado mental & $\leq 12 \mathrm{~h}$ & de 12 a $24 \mathrm{~h}$ & $>24 \mathrm{~h}$ \\
\hline Amnésia pós-traumática & de 1 min a $12 \mathrm{~h}$ & de $12 \mathrm{~h}$ a 3 dias & $<3>7$ dias \\
\hline Sintomas nas primeiras 12 horas & $1-2$ & $3-5$ & $>5$ \\
\hline Escala de coma de Glasgow & $13-15$ & $9-12$ & $<9$ \\
\hline
\end{tabular}

Abreviatura: TCE, traumatismo cranioencefálico.

Nota: Para classificarmos o grau do TCE apenas um critério deve estar presente, mesmo que os outros critérios contemplem um grau abaixo.

Tabela adptada de VA/DoD (2009). ${ }^{10}$ 
Traumatismo cranioencefálico leve Teixeira et al.

Tabela 5 Categorias de diagnóstico subagudo e crônico dos TCE

\begin{tabular}{|c|c|c|c|}
\hline Persistência & Grau 1 & Grau 2 & Grau 3 \\
\hline $\begin{array}{l}\text { Subagudo: permanência de } \\
\text { pelo menos um sintoma } \\
\text { pós-agudo de TCE por } 8-89 \\
\text { dias. Reavaliar o paciente } \\
\text { após } 15,30 \text { e } 60 \text { dias da } \\
\text { lesão. } \\
\text { Crônico: Permanência de } \\
\text { pelo menos um sintoma } \\
\text { pós-agudo do TCE por mais } \\
\text { de } 90 \text { dias. Reavaliar o } \\
\text { paciente após } 120 \text { dias, } 180 \\
\text { dias, } 1 \text { ano, } 1 \text { ano e meio e } 2 \\
\text { anos. }\end{array}$ & $\begin{array}{l}\text { Persistência de alguma } \\
\text { disfunção neurocognitiva, } \\
\text { sintomas somáticos, ou } \\
\text { desordem psicológica que } \\
\text { não atrapalhe na ocupação } \\
\text { ou as atividades sociais. }\end{array}$ & $\begin{array}{l}\text { Sintomas que impedem o } \\
\text { paciente de participar } \\
\text { plenamente de sua ocupação } \\
\text { ou atividades sociais. }\end{array}$ & $\begin{array}{l}\text { Sintomas que impedem o } \\
\text { paciente de participar } \\
\text { plenamente ou totalmente } \\
\text { de sua ocupação ou } \\
\text { atividades sociais. }\end{array}$ \\
\hline
\end{tabular}

Abreviatura: TCE, traumatismo cranioencefálico.

Nota: Tabela copiada de Krainin et al (2011). ${ }^{3}$

diferentes formas para se referir à lesão cerebral. O termo TCE leve tem sido usado constantemente no lugar do termo concussão, embora a definição do TCE leve varie bastante. Nesta revisão, aceitaremos os dois termos como sinônimos, representando claramente um trauma leve, não penetrante, e lesão traumática com uma curta alteração das funções cerebrais. ${ }^{13}$ A anamnese bem estruturada é considerada um Gold Standard para o diagnóstico de TCE leve, embora seja impraticável para a vigilância da população em geral, e assim podemos notar que uma proporção considerável dos TCE leves não é diagnosticada. Por isso, há a necessidade de critérios adicionais para a classificação do TCE leve, além da escala de Glasgow. ${ }^{14}$

Este é um dos poucos distúrbios da medicina em que a classificação benigna é enganosa, dada a um paciente no momento da lesão, e ainda pode estar associada a complicações ao longo da vida. O termo leve acaba sendo enganoso para os pacientes, familiares e público em geral. Sem uma compreensão clínica do TCE leve, o paciente pode voltar a atividades antes mesmo de estar totalmente recuperado. ${ }^{3}$

Devido a esta vasta gama de compreensão e a uma heterogeneidade na classificação, há um impacto negativo na interpretação e na comparação de achados no trauma leve (além de muitos estudos excluírem as concussões no esporte). Segundo uma revisão da Organização Mundial de Saúde (OMS), 62\% dos TCE leves são avaliados utilizando-se a escala de Glasgow num espectro variado que vai de 13 a 15; algumas classificações não incluem a perda da consciência e a amnésia, apesar de esses estudos apresentarem uma variação no tempo da alteração de consciência; já os estudos que não usam a escala de Glasgow como forma de classificação irão fazer uso da perda da consciência, ou da amnésia pós-traumática, ou de ambas. ${ }^{15}$ Outros estudos mostram que muitos centros fazem uso da nona revisão da Classificação Estatística Internacional de Doenças e Problemas Relacionados com a Saúde (CID-9) como forma de diagnóstico. Porém, ela tem se mostrado de baixa sensibilidade para classificar oTCE leve. Um estudo feito no contexto de salas de emergência mostrou que essa classificação, quando usada para vigilância da população, apresenta $98 \%$ de especificidade, e somente $46 \%$ de sensibilidade, sendo o valor preditivo negativo 99\%, e o valor preditivo positivo $24 \% .^{13}$ A CID-9 é a classificação mais frequentemente usada para se identificar o TCE leve, apesar da presença de falsos negativos e falsos positivos. Um levantamento mostrou que apenas $23 \%$ dos TCEs leves são classificados com a CID-9, além de $13 \%$ dos casos severos e $29 \%$ dos casos moderados. Por fim, existem alguns estudos que fazem uso da AIS como ferramenta de diagnóstico ou uma miscelânea entre estas ferramentas de diagnóstico. Esta variedade no uso de meios de classificação do TCE leve pode causar problemas ou erros de classificação. ${ }^{15}$ Uma preocupação notável com relação à definição DoD e VA para TCE leve é que, além de incluir Perda da Consciência e Amnésia Póstraumática, falta-lhe principais critérios no que diz respeito aos sintomas somáticos de curto e longo prazo, deficiências cognitivas e funcionais, e o tempo de duração dos sintomas. ${ }^{3}$

Não há um consenso quanto à classificação da gravidade do TCE. Há estudos que não verificaram alterações no exame de imagem associadas à dificuldade de aprendizado e de memória, assim como estudos que mostram exames com alterações sem apresentação de sintomas de um trauma leve. ${ }^{13}$ Devemos lembrar ainda que há uma dificuldade em definir sintomas posteriores à lesão, pois os mesmos são comumente associados a muitas outras causas, assim como o uso de medicamentos também pode alterar os testes de rastreamento. E, por fim, como outras dificuldades, podemos incluir sintomas clínicos que não são claros devido a sua sutileza ou ao atraso das manifestações. ${ }^{3}$

Para chegarmos a um consenso quanto à classificação dos TCE leves, e reconhecendo uma falta de unificação nesta classificação, a International Collaboration on Mild Traumatic Brain Injury Prognosis tem recomendado o uso da definição de TCE leve do American Congress of Rehabilitation Medicine (ACRM), que foi revisada pela WHO Collaborating Centre Task Force on Mild Traumatic Brain Injury (doravante, a força-tarefa da Organização Mundial de Saúde [OMS]). A definição de TCE leve do ACRM trata de uma alteração fisiológica das funções cerebrais, induzida por um trauma, resultado de uma pancada na cabeça por um objeto ou de a cabeça atingir um objeto, ou de o cérebro sofrer um 
movimento de aceleração ou desaceleração, seguida por pelo menos umas das seguintes manifestações: qualquer período de perda de consciência de até 30 minutos; amnésia póstraumática não superior a 24 horas; qualquer período de confusão ou desorientação; anormalidades neurológicas transitórias, incluindo sinais focais, convulsões, e lesão intracraniana que não requer cirurgia; escala de Glasgow entre os valores 13 e 15 (ou seja, que vão desde a confusão à consciência normal no exame dentro de 30 minutos após a apresentação). A força-tarefa da OMS estipulou que nenhuma dessas manifestações pode ser devido ao álcool, drogas, medicamentos, doença sistêmica, ou ferimento extracraniano. No entanto, o ACRM e a Força-Tarefa da OMS não especificou períodos mínimos de perda de consciência, amnésia pós-traumática, e desorientação, nem especificam como diferenciar o estresse importante após um evento traumático de concussão devido a um trauma na cabeça. Servadei e colegas definem o TCE leve restrita a pontuação GCS para 14-15, porque os resultados de pacientes com uma pontuação GCS de 13 são mais semelhantes aos do TCE moderado. ${ }^{4}$

Com relação à fisiopatologia do TCE leve, a lesão ocorre em duas fases, que se dão em tempos diferentes após a lesão. A primeira fase diz respeito à lesão direta, que pode resultar em uma lesão axonal difusa, hemorragia, laceração ou uma contusão cerebral direta causada por forças externas. Já a segunda fase ocorre algum tempo após a lesão, e envolve isquemia, edema, disfunção metabólica e inflamação. A segunda fase da lesão costuma ser aparente em exames de imagem (como a isquemia e o edema cerebral); porém, as alterações metabólicas se apresentam como um desafio para o diagnóstico. Estas alterações causadas na segunda fase são difíceis de serem avaliadas no contexto da lesão aguda, pois ocorrem certo tempo após a lesão, assim como nos casos de politraumas, pois as avaliações da vitalidade do paciente são de maior importância. ${ }^{3}$

Apesar do termo "leve", ferimentos na cabeça e no cérebro induzem mudanças bioquímicas que ocorrem em nível celular. Estas alterações podem afetar o desempenho, a viabilidade e a recuperação de estruturas cerebrais e de funcionamento após uma lesão. ${ }^{16}$ As alterações bioquímicas subjacentes que ocorrem com TCE leve incluem uma cascata neurometabólica póslesão e alterações na neurotransmissão. $O$ aumento do potássio extracelular minutos após a lesão pode levar a uma onda de excitação ou depressão. A onda de depressão neuronal se manifesta como perda temporária da consciência, amnésia, ou outros déficits cognitivos. ${ }^{17}$ Durante a fase aguda do TCE leve, alterações nos neurotransmissores ocorrem quando percursos colinérgicos interrompidos causam alterações de consciência, que não estão relacionados com qualquer disfunção do sistema de ativação reticular. ${ }^{18}$ Por fim, a lesão axonal difusa pode ter uma apresentação sintomatológica bem diferenciada, pois ela pode atingir diversas áreas do cérebro.

\section{Epidemiologia}

Estima-se que os TCE leves representem cerca de $80-90 \%$ de todos os casos de traumas quando consideramos as populações civil e militar. Devido a esta alta incidência e prevalência dos traumas leves na população, temos como consequência fardos sociais e econômicos. Em um estudo de 1985, Max et al concluíram que $44 \%$ de todos os gastos relacionados aos TCE são devidos a traumas leves. ${ }^{4}$ Outros estudos, realizados com populações militares, apontam uma incidência de $8-22 \%$ em soldados expostos a operações de combate. Devido a esta falta de consenso para classificar oTCE leve, dada a sua apresentação clinica variável, os médicos continuam a ter dificuldade em avaliar com precisão e diagnosticar o TCE leve. ${ }^{3}$

Se considerarmos todos os TCE da população civil, 75\% são classificados como leves, sendo que a principal causa são as quedas de pessoas mais jovens do que 15 anos ou mais velhas do que 65 anos. Já em adolescentes e adultos jovens (15-35 anos), os principais motivos são os acidentes com automóveis, que representam $17 \%$ dos traumas leves, e os assaltos, que representem 10\% dos casos. Segundo o CDC, há aproximadamente 500 casos por 100 mil pessoas, mas estas taxas variam muito quando comparamos as faixas de idade e o sexo. Já com relação à incidência cumulativa durante a vida, ela é difícil de estimar, devido à grande gama de classificação dos TCE leves. ${ }^{13}$ Pode haver também diferenças geográficas na incidência do TCE, mas essas diferenças precisam ser vistas com cautela, dadas as metodologias díspares e a ampla gama de resultados relatados (20 a 790 TCE leves por 100 mil pessoas/ano). Esta diferença de valores se dá pelo fato de que países que apresentam baixas taxas de incidência relataram apenas traumas que envolvem tratamento cirúrgico; por outro lado, em países com rendas baixas e médias, observa-se um aumento da taxa de acidentes com veículos. ${ }^{14}$

Na análise dos dados das populações militares, há um paradoxo entre a severidade dos traumas e os sintomas associados e os relatos, e isto se deve ao fato de uma possível perda de memória momentânea no momento da lesão ou do acidente. Uma solução é tentar averiguar os dados, o histórico e os sintomas do paciente o mais próximo quanto possível do momento da lesão, como nos registros de trauma. No entanto, os registros de trauma também têm limitações. Uma revisão do UK Joint Theater Trauma Registry, por exemplo, descobriu que 19\% dos ferimentos de batalha registrados eram TCE, a maioria (86\%) dos quais de moderados a graves, com apenas $39 \%$ dos casos sobrevivendo ao TCE. ${ }^{19}$ Nesta população, observam-se os seguintes mecanismos de trauma: acidentes representam $74 \%$ dos casos, e tentativas de agressão representam $11 \%$. Entre os acidentes, há acidentes de trânsito (20\%), quedas (20\%), e casos em que o paciente foi atingido por um objeto (15\%). Apesar dos resultados, estima-se que os casos militares não relatados são quatro vezes maiores se comparados aos casos documentados, por isso não devemos confiar muito nos dados. ${ }^{13}$

Estima-se que nos Estados Unidos os relatos anuais de concussões no esporte variem entre 1,6 e 3,8 milhões de casos novos, sendo que estes números podem chegar a representar cerca de $30 \%$ de todas as concussões que ocorrem em jovens. Novamente, esta diferença na análise de dados se refere às atuais limitações em estimar as concussões, devido à variedade de classificações e à ausência de relatos de muitos casos de concussão, principalmente se considerarmos a população 
de atletas. ${ }^{12}$ Devido a estas limitações, a frequência de concussões relacionadas ao esporte pode ser expressa pela criação de uma base de dados de exposição do atleta (EA). Uma EA é definida pelo registro da participação de um atleta, seja em um treino ou em uma competição (todo evento deve ser relatado). A incidência de concussão em atletas do Ensino Médio americano tende a ser entre 0,23 a 0,51 por $1.000 \mathrm{EAs}$, ao longo dos 7 anos acadêmicos, de acordo com a National High School Sports - Re. Com relação ao sexo, é difícil determinarmos a frequência para todos os esportes, pois há uma variedade grande de atletas de ambos os sexos segundo cada esporte, mas podemos encontrar uma relação importante entre um determinado tipo de esporte e o risco de concussão. ${ }^{20}$

Se considerarmos agora os atletas mais jovens, com idades entre 8 e 12 anos, iremos encontrar uma incidência de 6,16 por 1.000 EAs durante as competições, e de 0,24 por 1.000 EAs durante os treinos. Outra investigação de monitoramento das forças biomecânicas em atletas identificou 1,5 impactos na cabeça a cada treino, enquanto a cada competição foram identificados 3,7 impactos. Essas investigações indicam claramente que o momento da competição acaba sendo de risco maior para os atletas. Ainda poderemos encontrar diferenças nos achados em atletas quando compararmos os resultados advindos de países de altitudes diferentes. Isto se deve a um aumento da pressão intracraniana (PIC) em uma resposta à hipóxia (em países de alta altitude), e este aumento acaba sendo um fator protetor para concussões em casos de impacto na cabeça, pois impede que o cérebro se movimente no interior da caixa craniana (como no caso de golpe/contragolpe). ${ }^{13}$

Esta dificuldade chegar a conclusões a respeito dos TCEs leves se deve à heterogeneidade na apresentação dos estudos. Além disso, muitas pesquisas usam como base de dados para calcular a incidência de casos a CID-9, que, como já vimos, apresenta diversos problemas na classificação da gravidade do TCE. Por isso, nas pesquisas futuras, é preciso considerar essa variedade de classificação para melhor entendermos a incidência real dos TCE leves. ${ }^{15}$

\section{Diagnóstico}

Segundo Krainin et al:

"A International Collaboration on Mild Traumatic Brain Injury Prognosis tem recomendado o uso da definição de TCE leve do American Congress of Rehabilitation Medicine (ACRM), que foi revisado pela força-tarefa da OMS. A definição de TCE leve segundo o ACRM diz respeito a uma alteração fisiológica das funções cerebrais, induzida por um trauma, resultado de uma pancada na cabeça por um objeto, ou por um movimento de aceleração/desaceleração do cérebro no interior da caixa craniana, seguido por pelo menos umas das seguintes manifestações: período de perda de consciência de até 30 minutos; amnésia pós-traumática não superior a 24 horas; qualquer período de confusão ou desorientação; anormalidades neurológicas transitórias, incluindo sinais focais, convulsões, e lesão intracraniana que não requer cirurgia; escala de Glasgow entre os valores 13 e 15 (ou seja, que vão desde a confusão à consciência normal no exame dentro de 30 minutos após a apresentação). A força-tarefa da OMS estipulou que nenhuma destas manifestações pode ser devido ao álcool, às drogas, aos medicamentos, à doença sistêmica, ou ao ferimento extracraniano." ${ }^{3}$ poderemos diagnosticar, segundo a gravidade dos sintomas, em TCE leve. Já com relação aos sintomas pós-concussão, eles são comuns no trauma leve. Entre os sintomas, podemos incluir: sintomas físicos devido à lesão cerebral (náusea, tontura, dor de cabeça, visão turva, cansaço, e distúrbios do sono que não podem ser explicados por outro tipo de lesão periférica); déficits cognitivos (memória ruim, déficit de atenção e das funções executivas) que não podem ser explicados por um estado emocional ou por outras causas; e alterações comportamentais e emocionais (depressão, irritabilidade, ansiedade relatada, e labilidade) que não podem ser explicadas por uma reação psicológica devido a um estresse emocional, físico ou por outras causas. Porém, muitos centros médicos não utilizam os sintomas pós-concussão para diagnosticar o TCE leve. ${ }^{4}$

O TCE é uma condição médica grave que deve ser controlada e acompanhada em todos seus estágios: agudo, subagudo e crônico, mas devemos ter uma maior atenção ao manejo agudo do TCE leve. Sendo assim, em levantamentos primários, feitos por meio de entrevistas pós-lesão, podem não constar sintomas que se desenvolvem posteriormente e, dessa forma, o médico deve orientar o paciente para que se submeta a avaliações adicionais e, se necessário, repetir exames, se os sintomas atuais persistirem ou se novos sintomas surgirem. ${ }^{3}$

A respeito da avaliação doTCE leve, há algumas ferramentas que podem ser utilizadas, porém cada uma delas deve ser usada em um contexto diferente. Primeiramente, a Military Acute Concussion Evaluation (MACE) costuma ser uma ferramenta suplementar de avaliação, e os especialistas orientam que ela deve ser utilizada num período de 24 a 72 horas após a lesão. Foram reportadas algumas dificuldades com relação a esta ferramenta de avaliação como baixa sensibilidade e um pequeno efeito prático. Além disso, existe uma baixa correlação entre MACE e a severidade do TCE. Outra ferramenta para avaliar o TCE leve é a Neurocognitive Assessment Tool (NCAT), que é mais eficiente na comparação e avaliação das funções neurocognitivas anteriores à lesão e após a lesão, e um ferramenta importante no acompanhamento e vigilância dos pacientes. Por fim, há também o Immediate Post-concussion Assessment Cognitive Tool (ImPACT), que foi adaptado para muitos esportes nos Estados Unidos. A metodologia utilizada para avaliar o TCE gera resultados de fácil interpretação, não havendo a necessidade da avaliação de especialistas das áreas de neurologia e psiquiatria. Sendo assim, este teste fornece uma resposta imediatamente após a sua realização, que pode ser claramente compreendida pelo paciente e por quem o acompanha. O ImPACT, além de facilitar o processo de avaliação dos TCE leves, promove novas tendências de análise a respeito do tema. ${ }^{3}$

As concussões antigamente eram concebidas como lesões puramente fisiológicas, resultado de uma disfunção metabólica do cérebro devido a: mudanças nos gradientes de íons; alterações nos canais de sódio, potássio e cálcio; e 
desregulação nos neurotransmissores e inflamação. ${ }^{17}$ Porém, estudos que utilizam técnicas de neuroimagem estrutural mostraram que a ressonância magnética (RM) identificou anormalidades estruturais muito sutis na substância branca e na microcirculação, sendo que estas foram encontradas em uma quantidade substancial de pacientes com TCE leve, principalmente naqueles que apresentaram sintomas pós-concussão, ou naqueles em que as incapacidades permaneceram por mais do que três meses após a lesão. Devido aos achados nos exames de neuroimagem, percebeu-se que o mecanismo patológico predominante é a lesão axonal difusa. Devido à sua apresentação sintomatológica variada, em alguns exames de imagem encontramos achados sem sintomatologia, enquanto em outros exames não encontramos nenhum achado, porém a sintomatologia apresentada pelo paciente pode ser moderada ou grave. Além disso, a RM pode ser usada para mensurar a difusão da água ao longo do eixo da substância branca (edema cerebral), e estas alterações podem ser identificadas após um determinado tempo (duas semanas). Ainda com relação aos exames de imagem, temos que considerar os achados de micro-hemorragias difusas (que normalmente estão associadas ao TCE grave), as anormalidades no fluxo sanguíneo cerebral, e a reatividade vascular cerebral, que podem ocorrer no TCE leve, principalmente em pacientes que sofreram múltiplos traumas leves ou que sofrem com a permanência dos sintomas. Os achados de neuroimagem a partir da RM usam a ponderação da imagem em T2, que é mais sensível a micro-hemorragias, permitindo identificar a lesão vascular difusa em $24 \%$ dos pacientes que apresentaram TCE leve no centro de trauma. ${ }^{4}$

Outras anormalidades encontradas no TCE leve usando a tomografia computadorizada (TC) incluem concussão local, hemorragia subaracnóidea traumática, e hematomas extraaxiais (que incluem os hematomas epidural e subdural). Como esperado, a RM mostrou mais sensibilidade na identi- ficação de anormalidades sutis. Estudos mostraram que 28\% dos pacientes presentes no centro de trauma, após um intervalo de 12 dias da lesão, e com TC negativa, apresentam um resultado de ressonância anormal. ${ }^{21}$ Pode-se usar ainda a eletroencefalografia quantitativa, que é muito usada para identificar disfunções psicológicas após o TCE leve, e mostra evidências da persistência da disfunção neuronal dias ou semanas após a resolução clínica dos sintomas.

Ao considerarmos as dificuldades em diagnosticar o TCE leve, verificamos que 65\% dos traumas leves apresentam um fator concomitante que dificulta sua avaliação e identificação. Os dados sugerem que pacientes com consumo de álcool recente apresentam, mais frequentemente, sinais e sintomas de TCE leve, sugerindo que o álcool mimetiza artificialmente tais sintomas. Devido a isso, este grupo apresenta maior prevalência de TCE leve. Sendo assim, a presença de fatores concomitantes é o principal motivo que dificulta o desenvolvimento e validação de métodos diagnósticos e procedimentos de manejo clínico para estes pacientes, pois os mesmos se apresentam como uma população heterogênea e frequentemente terão comorbidades relacionadas e fatores de confusão, sendo que estes possuem sintomatologia semelhante ao do TCE leve. Por isso, é de grande importância para os médicos saber a relação entre a heterogeneidade de apresentação do TCE leve e sua relação com comorbidades ou a sua sobreposição a outras doenças psiquiátricas. Tendo em vista isso, os fatores que complicam o diagnóstico e os estudos na área foram avaliados segundo a sua prevalência (-Fig. 1). Há numerosas condições neuropsiquiátricas, medicamentosas e de abuso de drogas que atrapalham a investigação do TCE leve. No caso de álcool e drogas, devemos esperar determinado tempo até que o seu efeito passe para avaliar o paciente. Cabe lembrar que muitas variáveis ainda não foram avaliadas, como raça, questões socioeconômicas, nível educacional e TC. ${ }^{22}$

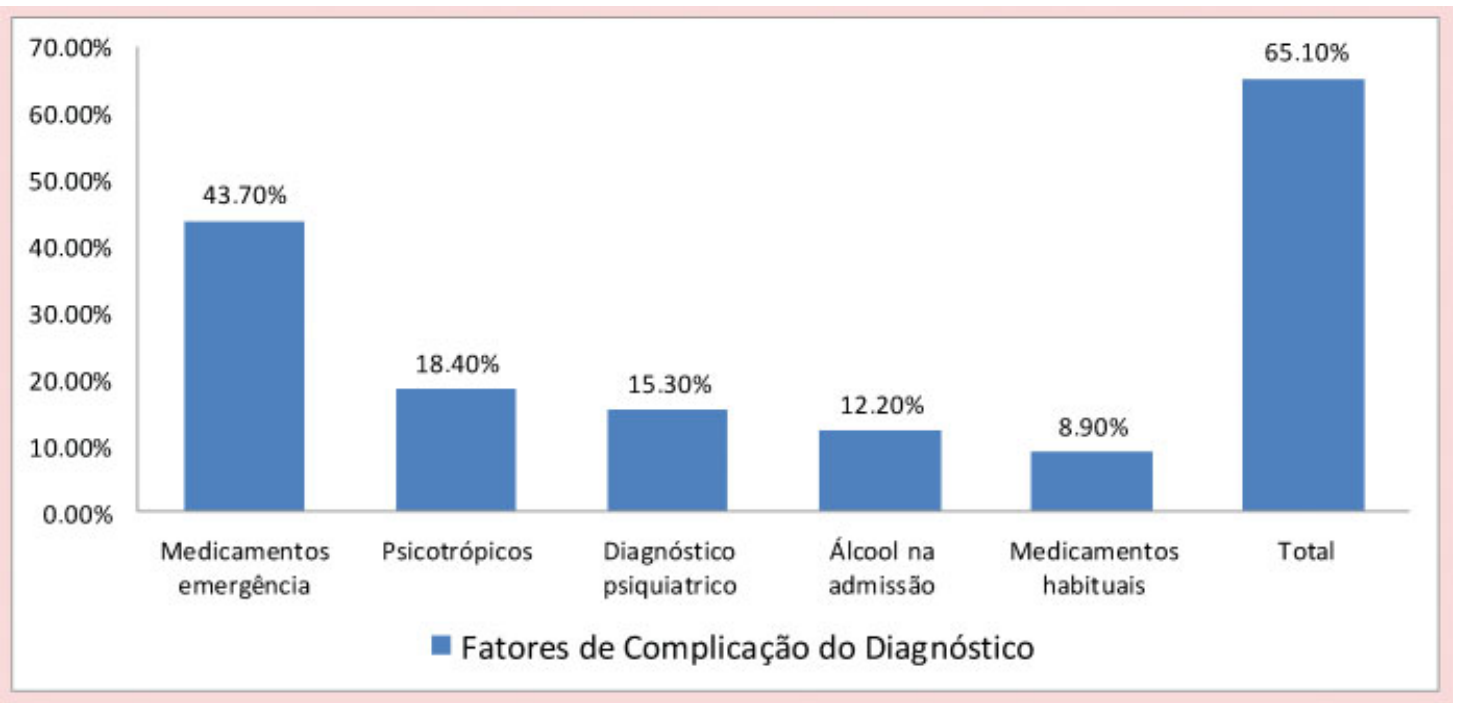

Fig. 1 Frequência de fatores complicadores em pacientes que apresentam um mecanismo comum de TCE leve (figura adaptada de Furger et al, 2015). ${ }^{22}$ 


\section{Prognóstico}

Os sintomas pós-concussão, incluindo sintomas emocionais (irritabilidade, depressão); sintomas físicos (dor de cabeça e tonturas); e sintomas cognitivos (memória e atenção ruins) se resolvem gradualmente na maioria dos pacientes com TCE leve dentro de aproximadamente 12 semanas, porém existem muitos pacientes em que esses sintomas se prolongam além desse período de três meses. No caso de o paciente apresentar um transtorno neuropsiquiátrico prévio, ele está fortemente relacionado à persistência dos sintomas por três meses ou mais. Já com relação aos atletas, este período de recuperação acaba sendo menor se comparado com a população em geral, sendo que atletas adultos e saudáveis têm um tempo médio de recuperação de 7-10 dias após a sua primeira concussão. Além disso, a perda de consciência, as comorbidades e os outros sintomas se apresentam de forma diferente nesta população, o que sugere que a concussão no esporte possa ser outro tipo de TCE leve. Devido a este possível prolongamento ou não dos sintomas, os sintomas subagudos se apresentam diferentes daqueles sentidos no momento do trauma. Sendo assim, foi proposta a troca do termo "sintomas pós-traumáticos" por "sintomas pósconcussão". 4

Constantemente, queixas como tristeza e cansaço aparecem em pacientes após três meses doTCE leve se comparados a controles saudáveis. Apesar de a recuperação funcional demorar cerca de três meses, pacientes com TCE leve frequentemente voltam ao trabalho no tempo médio de um mês ou mais, mas cerca de um terço deles acaba desempregado após três meses. Quando retornam ao trabalho, pacientes com TCE leve têm relatado que despendem mais esforços para realizar suas tarefas e se sentem mais cansados do que no momento anterior à lesão. ${ }^{4}$

Já com relação aos achados, prejuízo da memória, processamento lento das informações, e disfunção executiva são achados comuns em testes neuropsicológicos dentro da primeira e segunda semanas após a lesão. Déficits cognitivos que afetam a atenção, a velocidade de processamento e a memória são frequentemente presentes durante a primeira semana ou até um mês após a lesão. Estima-se que 80-85\% dos pacientes, dentro de 3-6 meses após um TCE leve isolado, se recupera dos sintomas iniciais. ${ }^{4}$ Achados patológicos em análises de imagens contribuíram para previsão de consequências em pacientes com escala de Glasgow de 13-15. Por exemplo, em um paciente com Glasgow de 13-15, com patologia intracraniana detectada por TC dentro de 24 horas, como lesões parenquimatosas, hemorragia intracraniana ou extracraniana, inchaço do cérebro, lesão de cisalhamento, e fraturas depressivas ou da região basilar, está associado com um pior prognóstico se comparado com pacientes que não tiveram patologia detectada no exame de TC. ${ }^{15}$

Muitos estudos sobre o TCE leve concordam que os sintomas, normalmente, melhoram entre uma semana e três meses, mas uma pequena porcentagem de pacientes acaba tendo recorrência desses sintomas por mais tempo. Algumas diretrizes para prática clínica a respeito de TCE sugerem que pacientes que tenham sintomas por mais de 30 dias após a concussão não devem estar sofrendo sintomas causados pela lesão, e que uma avaliação mais aprofundada se faz necessária para averiguar causas subjacentes. ${ }^{3}$ Um estudo de 2002 para validar o índice HeadMinder ${ }^{\circledR}$ de estabilidade cognitiva, um instrumento computadorizado, envolveu 23 casos de TCE leve ou moderado, não esportivo; nele, verificou-se uma média de 14 meses de recuperação após uma lesão. Este estudo encontrou em suas avaliações desempenho prejudicado na velocidade de processamento, no limite de velocidade de processamento, além de tempo de reação e memória abaixo da média. ${ }^{23}$ Um outro estudo de 2009 explorou a fisiopatologia da TCE leve, e observou que até $15 \%$ dos pacientes apresentaram sintomas até um ano após a concussão. Sendo assim, apesar de os estudos mostrarem a persistência dos sintomas por uma semana a três meses, os sintomas pósconcussão também podem permanecer por mais de um ano. Como o prognóstico no TCE leve pode diferir em um grande número de fatores, alguns autores afirmam que alguns fatores são mais fortes para indicar uma permanência dos sintomas, como os fatores psicológicos, o nível educacional do paciente, $\mathrm{e}$ persistentes compensações financeiras e litígios. Outros estudos discutem uma série de outros fatores que influenciam no prolongamento dos sintomas, como distúrbios mentais anteriores ou concomitantes, e falta de apoio social, que afeta negativamente os resultados. ${ }^{3}$

Os resultados clínicos mostram que os TCE leves têm uma curva de recuperação mais rápida quando comparados aos casos moderados e graves. Porém, em longo prazo, ainda podemos encontrar sintomas da lesão. Zumstein et al publicaram os resultados de seu estudo prospectivo de 10 anos, envolvendo 86 pacientes civis com TCE leve, que demonstrou que $37 \%$ dos pacientes relataram reduções significativas na sua qualidade de vida, medida pelo Beltz Scores. Além disso, a percentagem dos pacientes que sofriam de dores de cabeça aumentou de 11,4\% para 52,3\% entre 1 ano e 10 anos de após a lesão. Embora seus pacientes tenham voltado a trabalhar dentro de um ano, $11 \%$ relataram dificuldades ocupacionais após 10 anos. ${ }^{24}$ Por fim, há outros sintomas que acompanham a concussão, e que podem ter maior significado para a avaliação do TCE leve do que aqueles relacionados com a perda de consciência e a amnésia pós-traumática, como no caso das dores de cabeça e tonturas que se seguem à lesão, e estas ainda apresentam uma maior relação com recuperações em longo prazo. ${ }^{3}$

Por fim, com relação ao estresse pós-traumático, este se mostra muito mais prevalente nos TCE leves se comparados com os casos moderados e graves. O transtorno de estresse pós-traumático (TEPT) é consequência de uma exposição psicologicamente traumática e com risco de vida, acompanhada por uma resposta de medo, desespero ou pavor. Os sintomas, como re-experimentação, entorpecimento emocional, comportamento evasivo ou hiperexcitação podem permanecer ao longo de um mês, e resultam em prejuízo funcional significativo. Podemos verificar também que pacientes diagnosticados com TEPT e TCE leve costumam apresentar taxas maiores de sintomas pós-concussão e problemas psicossociais. Porém, no caso da lesão aguda, os sintomas da lesão e as deficiências neurocognitivas são mais 
proeminentes do que os sintomas psicológicos; sendo assim, estes podem passar despercebidos. Tais sintomas, como diminuição da função cognitiva e do humor, podem ser secundários a qualquer TCE, TEPT, ou a ambos, e cabe aos profissionais de saúde considerar cuidadosamente os sintomas psicológicos em seus diagnósticos diferenciais para não deixar passar nenhum caso de TEPT. Os sintomas psicológicos comuns que se sobrepõem ao TCE incluem irritabilidade, impulsividade, ansiedade, depressão, distúrbios do sono, transtornos por uso de substâncias psicoativas, e alterações de personalidade. Em última análise, prestadores de serviços médicos devem, cuidadosamente, verificar e considerar se a apresentação clínica do paciente se deve a uma lesão física ou a outras variáveis de confusão. ${ }^{3}$

\section{Referências}

1 Marshall LF, Marshall SB, Klauber MR, et al. The diagnosis of head injury requires a classification based on computed axial tomography. J Neurotrauma 1992;9(Suppl 1):S287-S292

2 Menon DK1, Schwab K, Wright DW, Maas AIDemographics and Clinical Assessment Working Group of the International and Interagency Initiative toward Common Data Elements for Research on Traumatic Brain Injury and Psychological Health. Position statement: Definition of traumatic brain injury. Arch Phys Med Rehabil 2010;91(11):1637-1640

3 Krainin BM, Forsten RD, Kotwal RS, Lutz RH, Guskiewicz KM. Mild traumatic brain injury literature review and proposed changes to classification. J Spec Oper Med 2011;11(03):38-47

4 Levin HS, Diaz-Arrastia RR. Diagnosis, prognosis, and clinical management of mild traumatic brain injury. Lancet Neurol 2015;14(05):506-517

5 Gaudêncio TG, Leão GM. A epidemiologia do traumatismo crânioencefálico: um levantamento bibliográfico no Brasil. Rev Neurocienc 2013;21(03):427-434

6 Adams H, Mitchell DE, Graham DI, Doyle D. Diffuse brain damage of immediate impact type. Its relationship to 'primary brain-stem damage' in head injury. Brain 1977;100(03):489-502

7 Kotapka MJ, Gennarelli TA, Graham DI, et al. Selective vulnerability of hippocampal neurons in acceleration-induced experimental head injury. J Neurotrauma 1991;8(04):247-258

8 Bigler ED. Anterior and middle cranial fossa in traumatic brain injury: relevant neuroanatomy and neuropathology in the study of neuropsychological outcome. Neuropsychology 2007;21(05): 515-531

9 Teasdale G, Jennett B. Assessment of coma and impaired consciousness: a practical scale. The Lancet 1974;304(7872):81-84

10 Department of Veterans Affairs and Department of Defense. The Management of Concussion/mTBI Working Group. (2009). VA/ DoD clinical practice guideline for management of concussion/ mild traumatic brain injury (mTBI). Accessed 20 July 2011 from (http://www.healthquality.va.gov/management_of_concussion_ mtbi.asp).

11 Brasure M, Lamberty GJ, Sayer NA, et al. Multidisciplinary postacute rehabilitation for moderate to severe traumatic brain injury in adults. Agency for Healthcare Research and Quality (AHRQ). AHRQ Comp Eff Rev 2012;72:ES1-ES20 Available at http://effectivehealthcare.ahrq.gov/ehc/products/283/1141/CER72_TBIPostacute_FinalReport_20120725.pdf

12 American Psychiatry Association et al. DSM-5: Manual diagnóstico e estatístico de transtornos mentais. São Paulo: Artmed Editora; 2014

13 Voss JD, Connolly J, Schwab KA, Scher AI. Update on the Epidemiology of Concussion/Mild Traumatic Brain Injury. Curr Pain Headache Rep 2015;19(07):32

14 Walker W, Cifu DX, Hudak A, Goldberg G, Kunz RD, Sima A. Structured interview for mild traumatic brain injury after military blast: interrater agreement and development of diagnostic algorithm. J Neurotrauma 2015;32:464-473

15 Carroll LJ, Cassidy JD, Holm L, Kraus J, Coronado VG; WHO Collaborating Centre Task Force on Mild Traumatic Brain Injury. Methodological issues and research recommendations for mild traumatic brain injury: the WHO Collaborating Centre Task Force on Mild Traumatic Brain Injury. J Rehabil Med 2004;36(43, Suppl) 113-125

16 Signoretti S, Vagnozzi R, Tavazzi B, Lazzarino G. Biochemical and neurochemical sequelae following mild traumatic brain injury: summary of experimental data and clinical implications. Neurosurg Focus 2010;29(05):E1

17 Giza CC, Hovda DA. The neurometabolic cascade of con-cussion. J Athl Train 2001;36(03):228-235

18 DeLellis SM, Kane S, Katz K. The neurometabolic cascade and implications of mTBI: mitigating risk to the SOF community. J Spec Oper Med 2009;9(04):36-42

19 Hawley CA, de Burgh HT, Russell RJ, Mead A. Traumatic brain injury recorded in the UK Joint Theatre Trauma Registry among the UK Armed Forces. J Head Trauma Rehabil 2015;30(01): E47-E56

20 Rosenthal JA, Foraker RE, Collins CL, Comstock RD. National high school athlete concussion rates from 2005-2006 to 2011-2012. Am J Sports Med 2014;42(07):1710-1715

21 Yuh EL, Mukherjee P, Lingsma HF, et al; TRACK-TBI Investigators. Magnetic resonance imaging improves 3-month outcome prediction in mild traumatic brain injury. Ann Neurol 2013;73(02): 224-235

22 Furger RE, Nelson LD, Brooke Lerner E, McCrea MA. Frequency of factors that complicate the identification of mild traumatic brain injury in level I trauma center patients. Concussion 2016;1(02): CNC11

23 Erlanger DM, Kaushik T, Broshek D, Freeman J, Feldman D, Festa J. Development and validation of a web-based screening tool for monitoring cognitive status. J Head Trauma Rehabil 2002;17(05): 458-476

24 Zumstein MA, Moser M, Mottini M, et al. Long-term outcome in patients with mild traumatic brain injury: a prospective observational study. J Trauma 2011;71(01):120-127 